\title{
Cost-effectiveness of shared pharmaceutical care for older patients: RESPECT trial findings \\ RESPECT Trial Team
}

\section{ABSTRACT}

\section{Background}

Pharmaceutical care serves as a collaborative model for medication review. Its use is advocated for older patients, although its cost-effectiveness is unknown. Although the accompanying article on clinical effectiveness from the RESPECT (Randomised Evaluation of Shared Prescribing for Elderly people in the Community over Time) trial finds no statistically significant impact on prescribing for older patients undergoing pharmaceutical care, economic evaluations are based on an estimation, rather than hypothesis testing.

Aim

To evaluate the cost-effectiveness of pharmaceutical care for older people compared with usual care, according to National Institute for Health and Clinical Excellence (NICE) reference case standards.

Methods

An economic evaluation was undertaken in which NICE reference case standards were applied to data collected in the RESPECT trial.

Results

On average, pharmaceutical care is estimated to cost an incremental $£ 10000$ per additional quality-adjusted life year (QALY). If the NHS's cost-effectiveness threshold is between $£ 20000$ and $£ 30000$ per extra QALY, then the results indicate that pharmaceutical care is cost-effective despite a lack of statistical significance to this effect. However, the statistical uncertainty surrounding the estimates implies that the probability that pharmaceutical care is not costeffective lies between 0.22 and 0.19 . Although results are not sensitive to assumptions about costs, they differ between subgroups: in patients aged $>75$ years pharmaceutical care appears more cost-effective for those who are younger or on fewer repeat medications.

\section{Conclusion}

Although pharmaceutical care is estimated to be costeffective in the UK, the results are uncertain and further research into its long-term benefits may be worthwhile.

\section{Keywords}

cost-effectiveness; health services for the aged;

medication therapy management; pharmaceutical care.

\section{INTRODUCTION}

The RESPECT (Randomised Evaluation of Shared Prescribing for Elderly people in the Community over Time) trial evaluated pharmaceutical care in the NHS. The design and clinical results of that trial have been reported in an accompanying article ${ }^{1}$ and, although it is reported that there were no statistically significant differences in outcomes, economic evaluation typically used estimation rather than hypothesis testing. This article, therefore, uses RESPECT trial data to estimate the mean cost-effectiveness of pharmaceutical care and looks at the uncertainty in that estimate.

C Bojke, MSc, senior research fellow; $Z$ Philips, $P h D$, honorary fellow; M Sculpher, PhD, professor; Centre for Health Economics, University of York. P Campion, PhD, emeritus professor of primary care medicine; Postgraduate Medical Institute, The University of Hull. H Chrystyn, PhD, head of pharmacy, University of Huddersfield. S Coulton, MSc, professor, Centre for Health Service Studies, University of Kent. B Cross, BSc, research associate; V Morton, MSc, research fellow; S Richmond, MSc, research fellow, Department of Health Sciences, University of York. A Farrin, MSc, director and principal statistician (Health Sciences), Clinical Trials Research Unit, University of Leeds. G Hill, BSc, professional development pharmacist, East Riding and Hull Local Pharmaceutical Committee, Hull. A Hilton, PhD, lecturer, Faculty of Health and Social Care, The University of Hull. J Miles, PhD, behavioural scientist, RAND Corporation, Santa Monica, California, US. I Russell, DSc, professor, School of Medicine, Swansea University. I Chi Kei Wong, PhD, professor, School of Pharmacy, University of London.

Address for correspondence

Chris Bojke, Centre for Health Economics, University of York, Heslington, York, YO10 5DD.

E-mail: cb23@york.ac.uk

Submitted: 15 December 2008; Editor's response: 25 May 2009; final acceptance: 10 November 2009.

(C)British Journal of General Practice.

This is the full-length article of an abridged version published in print. Cite this article as: Br J Gen Pract 2010; DOI: 10.3399/bjgp09X482312. 


\section{METHOD}

\section{Aim}

The aim was to evaluate the cost-effectiveness of pharmaceutical care relative to usual care for people aged $>75$ years, who were living at home and receiving repeat prescriptions for five or more drugs.

\section{Overview}

This evaluation takes the perspective of the UK's NHS. It estimates all identifiable costs to the NHS of pharmaceutical care, including community pharmacy costs. It adopts a time horizon of 12 months and presents costs in £ sterling at 2004-2005 prices: the last complete year of data collection. Consistent with the methods used by the National Institute for Health and Clinical Excellence (NICE), it estimates health outcomes in quality-adjusted life years (QALYs). ${ }^{2}$

\section{Design, participants, setting, and} pharmaceutical care: the intervention The methodology of the RESPECT trial has been outlined in the accompanying paper.

\section{Data collection: health outcomes}

In addition to the data collection as described in the clinical paper, ${ }^{1}$ participants also completed EQ-5D questionnaires at five points in time during the study: at recruitment; immediately before pharmaceutical care began; 3 and 12 months thereafter; and at the end of the study period, some 3 years after the start of recruitment.

The EQ-5D assesses five dimensions of health status: mobility, self-care, usual activities, pain or discomfort, and anxiety or depression. Participants' responses effectively locate them in a health state for which a 'utility' has been estimated from the preferences of 3400 members of the UK general population. ${ }^{3} \mathrm{~A}$ utility of one denotes perfect health, a utility of zero denotes 'as bad as death', and negative utilities identify states considered worse than death. As these utilities put a value on the quality of life associated with each health state, they can be used to weight duration of survival and, as such, estimate QALYs. The EQ-5D is NICE's preferred instrument for capturing utility in economic evaluations. ${ }^{4}$

\section{Resource use and costs}

Pharmacists recorded the time they spent with each participant in developing their pharmaceutical care plan and liaising with their GP. Information on each patient's health-service use between May 2001 and May 2005 was extracted from their GPs' records, thereby generating estimates of costs before, during, and after the intervention. These data included:

\section{How this fits in}

Evidence from the US and from hospital settings suggests that pharmaceutical

care may offer benefits to patients and save costs. In the UK both the Royal

Pharmaceutical Society of Great Britain and the UK Clinical Pharmacy

Association recommend the use of pharmaceutical care in the community. This,

the first UK-based randomised controlled trial to evaluate pharmaceutical care

in the community, indicates that that it is cost-effective, with $80 \%$ probability.

Further research into the long-term benefits of pharmaceutical care may be

worthwhile, particularly for a younger target population.

- drugs prescribed through acute and repeat prescriptions;

- laboratory tests ordered through primary care;

- visits to the general practice to see a GP or nurse, or to attend a GP or nurse-led primary care clinic;

- home visits and telephone consultations by GPs or nurses;

- inpatient admissions and length of stay; and

- outpatient visits.

The cost of these activities was estimated in $£$ sterling by deriving a unit cost for each item of resource use from routine sources..$^{5-8}$ Table 1 shows the main costs of health care, taken from an annually updated survey. ${ }^{5}$ For generic drugs the Drug Tariff was used at the time of analysis and, for branded drugs, the corresponding price list from Chemist and Druggist was used. ${ }^{7}$ The cost per item was then applied to the information recorded on the prescription, with regard to drug name, strength, and dose, and added a dispensing fee.

\section{Statistical methods}

The cost-effectiveness analysis was based on patients registered with general practices that used the Egton Medical Information Service (EMIS) electronic records system. This supported 633 of

\section{Table 1. Estimated healthcare costs.}

Item Unit cost, $£$

\begin{tabular}{ll}
\hline Cost per hour & 73 \\
GP & 33 \\
Community pharmacist & 26 \\
Locum pharmacist & \\
\hline Cost per consultation & 65 \\
GP home visit & 28 \\
GP clinic consultation & 24 \\
GP telephone consultation & 21 \\
GP surgery consultation & 20 \\
Nurse home visit & 9 \\
Nurse clinic consultation & 9 \\
Nurse telephone consultation & 9 \\
Nurse surgery consultation & \\
\hline
\end{tabular}




\begin{tabular}{|c|c|c|}
\hline & Mean & Standard deviation \\
\hline \multicolumn{3}{|l|}{ Initial visit } \\
\hline Consultation with participant/carer, minutes & 38.2 & 18.8 \\
\hline Liaison with GP, minutes & 5.4 & 12.5 \\
\hline $\begin{array}{l}\text { Time spent on pharmaceutical care before first } \\
\text { monitoring visit by participant, minutes }\end{array}$ & 28.8 & 42.4 \\
\hline Total time, minutes & 72.4 & 48.0 \\
\hline Fixed cost (including locum cover for all visits), $£$ & 62.90 & 47.00 \\
\hline Fixed cost (assuming no locum cover), $£$ & 46.30 & 42.70 \\
\hline \multicolumn{3}{|l|}{ Single typical monitoring visit } \\
\hline Consultation with participant/carer, minutes & 7.9 & 9.4 \\
\hline Liaison time with GP, minutes & 1.6 & 5.2 \\
\hline $\begin{array}{l}\text { Time spent on pharmaceutical care between } \\
\text { successive monitoring visits by participant, minutes }\end{array}$ & 5.7 & 12.6 \\
\hline Total time, minutes & 15.2 & 16.6 \\
\hline $\begin{array}{l}\text { Fixed cost/visit (including locum cover for } \\
\text { all consultations), } £\end{array}$ & 12.50 & 17.50 \\
\hline $\begin{array}{l}\text { Fixed cost/visit (including locum cover only } \\
\text { for home visits), } £\end{array}$ & 11.50 & 17.40 \\
\hline Fixed cost/visit (assuming no locum cover), $£$ & 10.20 & 15.10 \\
\hline
\end{tabular}

the 760 patients recruited into the study (83\%). Four patients withdrew before introduction of pharmaceutical care and were omitted from the analysis, so a total of 629 patients contributed to the survival probabilities. Thirty patients died during the control period; in accordance with the trial protocol, their cost and utility data did not contribute to the economic analysis. As such, 599 patients were involved in the analysis. However, it should be noted, that only 598 patients were used for utility analysis because one participant provided no EQ-5D data.

The analysis aimed to estimate the difference in mean costs and outcomes between an individual experiencing usual care and the same individual experiencing pharmaceutical care. As all living participants switched from the former to the latter, it was not possible to observe the whole of either pathway. Therefore, the required differences were estimated using a difference-in-difference econometric model..$^{9}$ This charts transition over time (with regard to the difference between start and finish) and the change in that transition due to the transfer from usual care to pharmaceutical care (regarding the difference in the difference between start and finish). Five parameters were estimated:

- survival;

- probability of a specified cost occurring;

- likely magnitude of specified cost given that it has occurred;

- probability of a utility of one occurring; and

- the likely difference from a utility of one, given that that difference has occurred.

The independent variables included in the analytical models, all defined a priori in the study protocol, were:

- patient's age, sex, and number of drugs on repeat prescription at the time of recruitment to the study;

- the time since recruitment to the study and the square of that time (to estimate non-linear transition over time);

- a binary variable representing the intervention; and

- the interaction between time and the intervention.

Stata software (version 8) was used to analyse survival by a parametric Weibull regression model, and estimated each of the other four parameters by a generalised linear model with the appropriate link function. To allow for the effects of time-invariant patient-specific factors, the generalised linear model used a multilevel random effects specification for measurements within patients within primary care trusts (PCTs)..$^{10}$

\section{Cost-effectiveness analysis}

These statistical analyses generated estimates of the differences in expected costs and QALYS between pharmaceutical care and usual care. In turn, this led to an incremental cost-effectiveness analysis averaged across participants in the study. ${ }^{11}$ If this were to estimate that pharmaceutical care (or

Table 3. Unadjusted monthly cost of health care by trial period.

\begin{tabular}{lcccc} 
& \multicolumn{3}{c}{ Mean (SD), $£$} \\
\cline { 2 - 5 } & $\begin{array}{c}\text { Pre-control } \\
\text { period }\end{array}$ & Control period & $\begin{array}{c}\text { Intervention } \\
\text { period }\end{array}$ & $\begin{array}{c}\text { Post-intervention } \\
\text { period }\end{array}$ \\
\hline Acute medications & $6.75(26.00)$ & $6.74(22.70)$ & $7.99(35.00)$ & $7.16(30.30)$ \\
\hline Repeat medications & $46.20(280.00)$ & $53.90(274.00)$ & $52.40(129.00)$ & $52.70(71.00)$ \\
\hline Laboratory tests & $3.13(14.69)$ & $3.47(15.24)$ & $4.50(17.51)$ & $4.36(17.42)$ \\
\hline Primary care & $21.50(39.60)$ & $19.30(34.20)$ & $23.50(41.70)$ & $23.00(46.70)$ \\
\hline Secondary care & $71.90(557.00)$ & $107.10(794.00)$ & $151.70(1252.00)$ & $132.90(991.00)$ \\
\hline Total & $149.00(643.00)$ & $190.00(1000.00)$ & $240.00(1179.00)$ & $220.00(1206.00)$ \\
\hline SD = standard deviation. & & & &
\end{tabular}


usual care) was less costly and more effective, it would 'dominate' the alternative. If not, the incremental cost-effectiveness ratio (ICER), the incremental cost per additional QALY, would be reported. To reflect uncertainty in the estimated costs and QALYs, the results of a Monte Carlo simulation were summarised in a QALY-cost plane and a cost-effectiveness acceptability curve that shows the simulated probability that pharmaceutical care is more cost-effective than usual care for a range of cost-effectiveness 'thresholds' - what the NHS might be willing to pay for an extra QALY.

\section{RESULTS}

\section{Estimated costs}

On average, pharmacists spent over 70 minutes per participant in setting up their pharmaceutical care plans (Table 2). This included time spent with the participant, time spent liaising with their GP, and time spent drafting the pharmaceutical care plan in isolation. Pharmacists also spent a mean of 15 minutes per patient monitoring visit. Depending on whether pharmacists used locums or overtime, this cost £46-63 per patient set up, and £10-13 per monitoring visit. This shows that most of the intervention costs occur at the beginning of care, when the pharmacist is setting up the care plan.

Table 3 shows that the unadjusted mean monthly costs of health care per patient show a general increasing trend over time, although this was not statistically significant. This may be a function of an underlying trend, as an already older trial population ages further, and/or may be the impact of the intervention. The statistical techniques explicitly attempt to separate these two potential effects.

\begin{tabular}{|c|c|c|c|c|}
\hline & $n$ & Mean & Median & SD \\
\hline Recruitment & 743 & 0.583 & 0.691 & 0.277 \\
\hline Baseline (before intervention) & 653 & 0.575 & 0.689 & 0.282 \\
\hline 3 months (during intervention) & 616 & 0.591 & 0.690 & 0.274 \\
\hline 12 months (end of intervention) & 558 & 0.601 & 0.691 & 0.264 \\
\hline Final (after intervention) & 533 & 0.589 & 0.689 & 0.271 \\
\hline
\end{tabular}

\section{Effectiveness}

Table 4 shows the unadjusted means, medians, and standard deviation of the EQ-5D derived utility scores per patient over the trial period. The raw unadjusted utilities show a decline from recruitment to baseline, a trend which is reversed when measured utility shows an increase at time points during the intervention. The final after-intervention score shows a decline from the final intervention value. As a natural decline over time is, perhaps, to be expected, this result is indicative of an effective intervention; however, as with costs, the model will show these potential effects.

\section{Cost-effectiveness}

Table 5 shows the modelled expectations for this population of patients over the observed period of time, thereby removing any confounding effect of time trends. On average, pharmaceutical care costs an estimated $£ 192$ a year more than usual care but yields slightly greater benefits, namely 0.019 of a QALY. Dividing the latter into the former, the incremental cost per additional QALY was estimated at $£ 10000$.

\begin{tabular}{|c|c|c|c|}
\hline Expectec & d population mean & $\begin{array}{c}\text { Simulated } \\
\text { 5th centile (lower limit) }\end{array}$ & $\begin{array}{l}\text { Simulated } \\
\text { 95th centile (upper limit) }\end{array}$ \\
\hline \multicolumn{4}{|l|}{ Cost analysis, $£$} \\
\hline Cost of usual care & 1809 & 1005 & 2484 \\
\hline Cost of pharmaceutical care & 2001 & 1109 & 2777 \\
\hline Incremental costs & 192 & -150 & 579 \\
\hline \multicolumn{4}{|l|}{ QALY analysis } \\
\hline QALYs in usual care & 0.595 & 0.402 & 0.736 \\
\hline QALYs in pharmaceutical care & 0.614 & 0.405 & 0.790 \\
\hline Incremental QALYs & 0.019 & -0.023 & 0.102 \\
\hline Incremental cost effectiveness ratio, $£$ & 10000 & $\begin{array}{l}\text { Pharmaceutical care } \\
\text { dominates usual care }\end{array}$ & $\begin{array}{l}\text { Usual care dominates } \\
\text { pharmaceutical care }\end{array}$ \\
\hline
\end{tabular}

QALY = quality-adjusted life year.

Note: These statistics arise from Figure 1. More than $5 \%$ of the simulated scenarios show higher costs of pharmaceutical care and fewer QALYS (those points lying in the northwest quadrant of the QALY-cost plane in Figure 1). In contrast more than $5 \%$ of simulated scenarios show lower costs of pharmaceutical care and more QALYs (those points lying in the southeast quadrant of the QALY-cost plane in Figure 1). Hence, the last two columns of Table 5 report the qualitative interpretation of these results - that the $90 \%$ credible interval includes both extremes, namely where pharmaceutical care dominates usual care and usual care dominates pharmaceutical care. 
Figure 1. Monte Carlo simulation of costs and QALYs and cost-effectiveness acceptability curve.
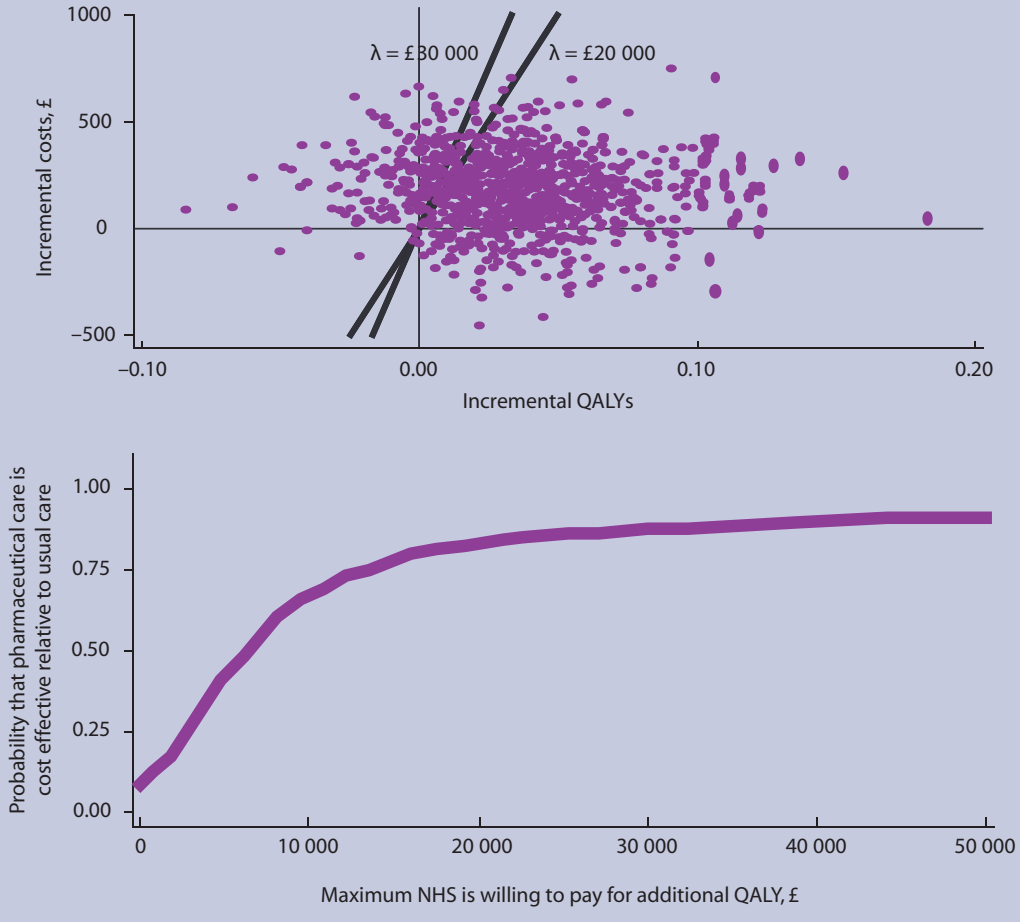

\section{Uncertainty and heterogeneity}

The reported expectations were based on coefficients estimated by the survival, utility, and cost-regression models. Although unbiased, these estimates exhibit uncertainty. To take this uncertainty into account, Table 5 reports the upper and lower credible intervals based on a probabilistic sensitivity analysis conducted using Monte Carlo simulation, with alternative parameters derived from the regression models' variance-covariance matrices.

Figure 1 further expresses the uncertainty by plotting the paired costs and benefits on a QALY-cost plane and the corresponding simulated probability that pharmaceutical care is more costeffective than usual care in a cost-effectiveness acceptability curve. As this threshold increases from zero to infinity, the probability that pharmaceutical care is more cost-effective than usual care increases from 0.10 and converges on 0.85 . At a threshold between $£ 20000$ and $£ 30000$ per QALY (as is consistent with NICE's threshold range), ${ }^{4}$ the probability that pharmaceutical care is cost-effective lies between 0.775 and 0.812 .

Several scenario analyses were undertaken to test the effect of alternative assumptions about costs on the results. These included alternative methods of costing visits, depending on the effect on pharmacies of patient non-attendance and whether they used locums. None of these analyses markedly changed either the average ICER or the probability of costeffectiveness.

The modelling approach also permits the characterisation of systematic variation between types of patient. Table 6 shows how cost-effectiveness varies between patients of different ages and differing numbers of repeat drugs and Figure 2 shows how expected utility varies between patients of different ages and differing numbers of repeat drugs. On average, pharmaceutical care is more cost-effective for younger patients on fewer drugs as, although the older

Table 6. Modelled costs and QALYs over 12 months for patient types under different methods of care.

\begin{tabular}{|c|c|c|c|c|c|c|c|}
\hline \multirow{2}{*}{$\begin{array}{l}\text { Modelled expectation for average } \\
\text { patient type }\end{array}$} & \multicolumn{3}{|c|}{ QALYs } & \multicolumn{3}{|c|}{ Costs, $£$} & \multirow{2}{*}{$\begin{array}{l}\text { ICER } \\
(£ / Q A L Y)\end{array}$} \\
\hline & Usual & PC & Difference & Usual & PC & Difference & \\
\hline 75 year old with 5 repeat drugs & 0.661 & 0.680 & 0.019 & 991 & 1080 & 89 & $£ 4661$ \\
\hline 80 year old with 7 repeat drugs & 0.604 & 0.620 & 0.016 & 1411 & 1566 & 155 & $£ 9515$ \\
\hline 85 year old with 10 repeat drugs & 0.520 & 0.537 & 0.017 & 2207 & 2510 & 302 & $£ 17980$ \\
\hline 90 year old with 15 repeat drugs & 0.363 & 0.383 & 0.020 & 4274 & 4977 & 703 & $£ 35185$ \\
\hline
\end{tabular}

ICER = incremental cost-effectiveness ratio. $P C=$ pharmaceutical care. QALY = quality-adjusted life year. 


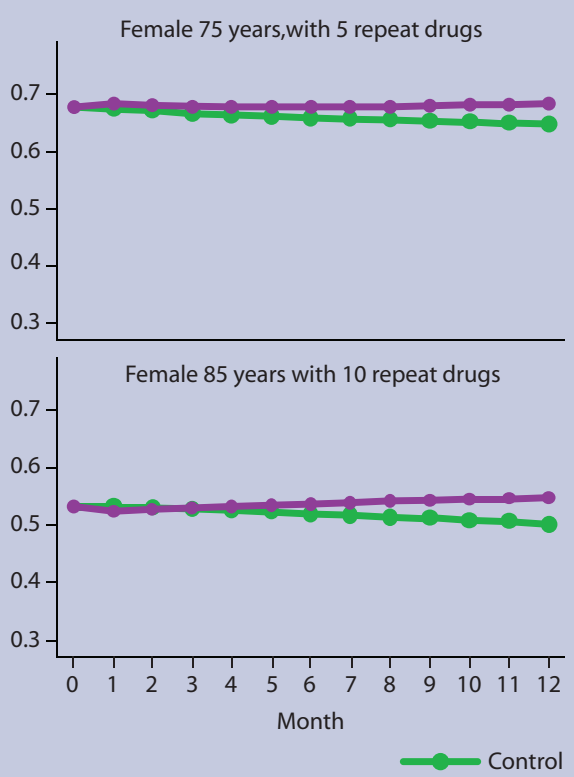

patient group are expected to have a similar - and possibly marginally higher - QALY gain, there are incremental costs for the younger patient group that are relatively significantly lower, leading to a lower ICER.

\section{DISCUSSION}

\section{Summary of main findings}

RESPECT is the first study to evaluate the costeffectiveness of community-based pharmaceutical care for older patients in the UK. Over 12 months, the pharmaceutical care intervention cost £192 more per patient than the usual care model, but led to an average improvement of 0.019 QALYs. Together, these findings suggest that the RESPECT model of pharmaceutical care costs $£ 10000$ for every QALY it gains. In making decisions about value for money of treatments, NICE generally uses a threshold of between $£ 20000$ and $£ 30000$ per QALY. ${ }^{4}$ This suggests that, on average, pharmaceutical care is cost-effective. However, the uncertainty in differential costs and QALYs means that the probability of the intervention being cost-effective is between $78 \%$ and $81 \%$ for this threshold range.

\section{Strengths and limitations of the study}

This trial covered five PCTs and adhered closely to its published design: a randomised multiple interrupted time series, which provided a robust approach to evaluating this complex intervention. RESPECT also exceeded its recruitment targets, and kept attrition well below target.

However, this analysis covers only 12 months, reflecting the length of the intervention within each PCT. Given the potential for much longer use of

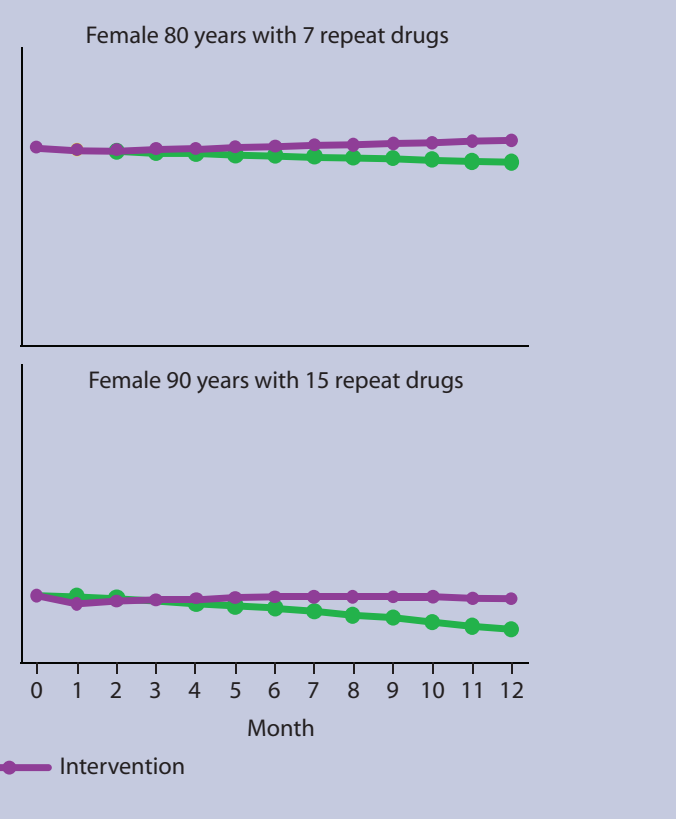

Figure 2. Modelled expected utility over time for average patient type. pharmaceutical care, it is important to consider how costs and effects might change over time. The data suggest that, after 12 months, the QALY benefits of pharmaceutical care increase. As such, the truncated period of analysis may have underestimated the full benefits.

\section{Comparison with existing literature}

Although there is evidence that pharmaceutical care can benefit patients with specific diseases, ${ }^{12}$ few studies relate to community pharmacies. Moreover, although modelling studies have suggested that pharmaceutical care can achieve savings in specific populations, ${ }^{13-15}$ there has been little prospective data collection in older people in the UK. ${ }^{16}$ The PEER study, the only study to assess benefits and costs of pharmaceutical care, which recruited some UK patients, ${ }^{17}$ suggested that such care had a small effect on health-related quality of life and achieved small cost savings. ${ }^{18}$

\section{Implications for future research and clinical practice}

This study's findings resemble those of the PEER study $;{ }^{17}$ however, there is uncertainty in the results of both studies. The differences in benefits and costs between intervention and usual care are small and do not always reach conventional levels of statistical significance. Although hypothesis tests underpin the clinical analysis of RESPECT, ${ }^{1}$ there are good reasons not to use statistical inference as a basis for decision making about resource allocation in health care; ${ }^{19}$ this is the general approach for economic analysis supported by NICE. However, policymakers should be aware of the uncertainty associated with 
encouraging the use of pharmaceutical care. Although the intervention is more cost-effective than usual care, on average, the probability of costeffectiveness only reaches approximately $80 \%$.

RESPECT evaluated pharmaceutical care for participants aged over 75 years who were on at least five 'repeat' drugs. The analytical approach implies that the cost-effectiveness differs across subgroups. For example, in comparing younger and older patients, the expected costs are lower in the younger group for a similar benefit gain. As such, if pharmaceutical care were to be implemented across the UK, it is possible that it may positively affect a population group for whom the cost-effectiveness is actually better than those observed in the trial that had a strictly enforced inclusion criteria.

Pharmaceutical care generates modest extra cost and health benefits compared with usual care. The mean incremental cost per QALY gained suggests that it may be cost-effective. However, there is considerable uncertainty in these findings. Further research may represent good value for money, not least to identify the long-term pattern of QALYS gained, potentially within RESPECT participants.

\section{Funding}

Research costs: Medical Research Council (grant reference G0001150). Excess treatment costs: Eastern Hull Primary Care Trust, East Yorkshire Primary Care Trust, Selby \& York Primary Care Trust, West Hull Primary Care Trust, and Yorkshire Wolds \& Coast Primary Care Trust

\section{Ethical approval}

Hull \& East Riding Research Ethics Committee (reference 06/01/107). York Research Ethics Committee, (reference 02/05/005)

\section{Competing interest}

The authors have stated that there are none

\section{Acknowledgments}

We thank Hilary Edmondson, Karen Goodyear and Andrew Hersom for support with the grant application; the Medical Research Council and primary care trust for funding; all health professionals and patients who participated in the research; Professor Lewis Ritchie (University of Aberdeen), Professor Alain Li Wan Po (University of Aston), and Dr Susan Ambler (Royal Pharmaceutical Society of Great Britain) for serving on the trial steering committee; Professor David Torgerson (Director of York Trials Unit, University of York) for academic support; all who contributed to developing the UK Medication Appropriateness Index; 15 undergraduate pharmacy students from Bradford School of Pharmacy who assisted with data collection; and Elizabeth Jones (University of Hull), Bee Lian Sim (University of London), and Valerie Wadsworth (University of York) for secretarial support. Ian Chi Kei Wong's post was funded by a Department of Health Public Career Scientist Award at the time of the study.

\section{Authors' contributions}

Chris Bojke has been trial economist since 2005. He contributed to the management, validation, analysis and interpretation of data, and writing this paper. He serves as the corresponding author for this paper following its publication.

lan Wong was grantholder and chief investigator responsible for the pharmacy component of the trial. He contributed to the original grant application, and the design, implementation and management of the trial. He guarantees the clinical content of this paper.
All authors commented on successive drafts of this paper, and can defend its content.

Mark Sculpher was principal investigator responsible for economic evaluation. He contributed to the economic protocol, interpreting data and writing this paper. $\mathrm{He}$ guarantees its economic content.

Ian Russell was grantholder and principal investigator responsible for trial methods. He contributed to the original grant application, the design and implementation of the trial, the economic protocol, interpreting data, and writing this paper. Stewart Richmond was trial manager, responsible for overall management and quality assurance. He contributed to the design, implementation, and management of the trial, developing procedures and data collection instruments, the design, implementation, and management of the recruitment and data collection plan, analysing and interpreting data, and writing this paper. He served as the corresponding author for this paper prior to its publication.

Zoë Philips was trial economist, responsible for designing and implementing the economic evaluation plan. She contributed to designing, implementing, and managing the trial, developing procedures and data collection instruments, and collecting, managing, analysing, and interpreting data. She prepared the initial draft of this paper.

Veronica Morton was trial statistician from December 2004, responsible for developing and implementing the statistical analysis plan, and validating data statistically. She contributed to the design, implementing and managing the trial, and managing, analysing, and interpreting data. Jeremy Miles was trial psychometrician responsible for the validity and reliability of questionnaires and data collection instruments. He contributed to the design and implementation of the trial, and the analysis and interpretation of data.

Graham Hill was pharmacy practice adviser. He contributed to the design, implementation, and management of pharmaceutical care.

Andrea Hilton was trial pharmacist, responsible for implementing and managing the introduction of, and training for, pharmaceutical care. She contributed to the design, implementation, and management of the trial, the anglicisation of the UK-MAI, developing and implementing procedures and data collection instruments.

Amanda Farrin was trial statistician until November 2004 and statistical adviser thereafter. She contributed to the design and implementation of the trial, developing data collection procedures and instruments, the statistical analysis plan, and the interpretation of results.

Ben Cross was assistant trial data manager. He contributed to database design and management.

Simon Coulton was trial data manager, responsible for database design and management. He contributed to the design and implementation of the trial, and developing questionnaires and data collection instruments.

Henry Chrystyn was academic pharmacy adviser. He contributed to the design, implementation and management of both the introduction of pharmaceutical care and the trial. Peter Campion was grantholder and principal investigator responsible for recruitment and training in primary care. He contributed to the original grant application, and the design, implementation and management of the trial.

\section{Discuss this article}

Contribute and read comments about this article on the Discussion Forum: http://www.rcgp.org.uk/bjgp-discuss

\section{REFERENCES}

1. RESPECT trial team. Effectiveness of shared pharmaceutical care for older patients: RESPECT trial findings. Br J Gen Pract 2009; DOI: 10.3399/bjgp09X473295

2. National Institute for Health and Clinical Excellence. Guide to the methods of technology appraisal. London: NICE, 2004.

3. Rabin R, Charro F. EQ-5D: a measure of health status from the EuroQol Group. Ann Med 2001; 33: 337-343.

4. National Institute for Health and Clinical Excellence. Guide to the methods of technology appraisal. London: NICE, 2008.

5. Curtis L, Netton A. Unit costs of health and social care. Canterbury: 
Personal Social Services Research Unit, University of Kent; 2005.

6. Surelines Pharmaceutical Services. Drug tariff. Blisworth: Surelines Ltd, 2005. http://www.drugtariff.com (accessed 19 Nov 2009).

7. Monthly price list - December 2005. Chemist and Druggist 2006; 46: 12.

8. Chartered Institute of Public Finance and Accountancy. Returns 2005. London: CIPFA; 2006.

9. Frolich M. Programme evaluation with multiple treatments. Journal of Economic Surveys 2004; 18: 181-224.

10. Skrondal A, Rabe-Hesketh S. Generalized latent variable modeling: multilevel, longitudinal and structural equation models. Boca Raton: Chapman and Hall, 2004.

11. Drummond MF, Sculpher M, Torrance G, et al. Methods for the economic evaluation of health care programmes. $3 \mathrm{rd}$ edn. Oxford: Oxford University Press, 2005.

12. Crealey GE, Sturgess IK, McElnay JC, Hughes CM. Pharmaceutical care programmes for the elderly: economic issues. Pharmacoeconomics 2003; 21: 455-465.

13. Bond C, Matheson C, Williams S, et al. Repeat prescribing: a role for community pharmacists in controlling and monitoring repeat prescriptions. Br J Gen Pract 2000; 50: 271-275.

14. Manley HJ, Carroll CA. The clinical and economic impact of pharmaceutical care in end stage renal disease patients. Semin Dia 2002; 15: 45-49.

15. Etemad L, Hay J. Cost-effectiveness analysis of pharmaceutical care in a Medicare drug benefit program. Value Health 2003; 6(4): 425-435.

16. Kennie NR, Schuster BG, Einarson TR. Critical analysis of the pharmaceutical care research literature. Ann Pharmacother 1998; 32: $17-26$

17. Bernsten C, Bjorkman I, Caramona M, et al. Improving the wellbeing of elderly patients via community pharmacy-based provision of pharmaceutical care: a multi-centre study in seven European countries. Drugs Aging 2001; 18: 63-77.

18. Sturgess IK, McElnay JC, Hughes CM, Crealey G. Community pharmacy based provision of pharmaceutical care to older patients. Pharm World Sci 2003; 25: 218-226.

19. Claxton K. The irrelevance of inference: a decision-making approach to the stochastic evaluation of health care technologies. Health Econ 1999; 18: 341-364. 